\title{
Características do Processo de Desmame da Ventilação Mecânica em Hospitais do Distrito Federal ${ }^{\star}$
}

\author{
Weaning from Mechanical Ventilation Process \\ at Hospitals in Federal District
}

\author{
Juliana Quixabeira Gonçalves', Raquel das Chagas Martins' ${ }^{1}$, Ana Paula Alves de Andrade², \\ Flavia Perassa de Faria Cardoso ${ }^{3}$, Maria do Horto Obes de Melo ${ }^{4}$
}

\section{RESUMO}

JUSTIFICATIVA E OBJETIVOS: O desmame da ventilação mecânica é um importante processo e rotineiro nas unidades de terapia intensiva (UTI). O objetivo deste estudo foi identificar, caracterizar e demonstrar as práticas utilizadas por fisioterapeutas respiratórios na obtenção dos parâmetros de desmame, bem como a sua execução em UTI.

MÉTODO: Foram incluídos somente fisioterapeutas atuantes em UTI no DF, no ano de 2005. Foi utilizado um questionário, composto por 31 perguntas, objetivas e subjetivas, relacionadas ao processo de desmame, sendo que algumas permitiam respostas múltiplas.

RESULTADOS: Foram avaliados 20 hospitais a partir

1. Fisioterapeuta Graduada pela Universidade Católica de Brasília (UCB).

2. Professora MSc e Supervisora do Estágio em Cárdio-Pneumologia do Curso de Fisioterapia da UCB; Orientadora de Trabalho de Conclusão de Curso de Fisioterapia da UCB; Fisioterapeuta da UTI do Hospital Universitário de Brasília -Universidade de Brasília (HUB - UnB).

3. Professora MSc e Supervisora de Estágio em Terapia Intensiva Adulto do Curso de Fisioterapia da UCB; Supervisora do Curso de Pós-Graduação em Fisioterapia Cardiorrespiratória da UCB.

4. Professora MSc e Supervisora do Estágio em Clínica Cirúrgica do Curso de Fisioterapia da UCB; Supervisora do Curso de Pós-graduação em Fisioterapia Cardiorrespiratória da UCB; Fisioterapeuta da UTI neonatal do Hospital da Ceilândia.

${ }^{*}$ Recebido do Departamento de Fisioterapia da Universidade Católica de Brasília (UCB - DF), Brasília, DF

Apresentado em 14 de dezembro de 2006.

Aceito para publicação em 23 de fevereiro de 2007

Endereço para correspondência:

Ana Paula Alves de Andrade.

SMT Conj 14 Casa 04 Taguatinga Sul

72023-400 Brasília, DF

Fone: (61) 9227-4138

E-mail: anapaulla@pop.com.br

(C)Associação de Medicina Intensiva Brasileira, 2007 de 80 questionários. Observou-se que $90 \%$ dos participantes apresentaram especialização na área de atuação, e, em média, tinham aproximadamente três anos de experiência em UTI. Médicos e fisioterapeutas, em $98,7 \%$ das respostas, são os profissionais responsáveis pelo manuseio dos ventiladores. Foi observado que $61,3 \%$ das respostas destacavam os médicos e fisioterapeutas em conjunto com os responsáveis pelo desmame e, 36,3\% somente o fisioterapeuta. Verificou-se que apenas 24 fisioterapeutas (30\%) seguem um protocolo para o desmame. Dentre os parâmetros rotineiramente avaliados no processo do desmame, a freqüência respiratória (98\%), o volume-corrente $(97,5 \%)$ e a saturação periférica de oxigênio $(92,5 \%)$ são os mais utilizados, sendo menos utilizados os índices de pressão inspiratória máxima $(18,8 \%)$ e a capacidade vital $(13,8 \%)$.

CONCLUSÕES: Observou-se grande variabilidade nos modos utilizados, na escolha dos parâmetros e na forma como foram coletados, sugerindo, então, a falta de rotina nos serviços e a necessidade de implantação de protocolos simples e facilmente aplicáveis.

Unitermos: desmame, fisioterapeutas, parâmetros, protocolo, ventilação mecânica

\section{SUMMARY}

BACKGROUND AND OBJECTIVES: Concerning the mechanical ventilation, the weaning is a usual and significant intensive care process. Identifying, describing and demonstrating the techniques used by Respiratory Therapists in weaning and also obtaining its parameters in Intensive Care Units (ICU).

METHODS: A survey related to the weaning process was done with active ICU Respiratory Therapists from (FD) in the year 2005. The survey consisted of 31 subjective and objective questions, some of them allowing multiple answers. 
RESULTS: Eighty surveys were carried out at twenty hospitals. $90 \%$ of participants were specialized staff with a mean of three year working experience in ICU. In $98.7 \%$ of the answers, doctors and respiratory therapists were responsible for operating the ventilators. In $61.3 \%$, doctors and respiratory therapists were responsible for their use and, in $36.3 \%$, the responsibility was solely on the respiratory therapist professionals. It was found that only twenty-four respiratory therapists $(30 \%)$ follow the weaning protocol. Among the most practiced parameters from the weaning process are: respiratory frequency $(98 \%)$, tidal volume $(97.5 \%)$ and periferic oxygen saturation $(92.5 \%)$. The least utilized are the maximum inspiratory pressure (18.8\%) and the vital capacity (13.8\%).

CONCLUSIONS: Great differences were observed in the weaning methods, choice of parameters and the way they were collected. These variations suggest that there is a lack of routine and the need to implement simple protocols.

Key Words: mechanical ventilation, parameters, protocol, respiratory therapists, weaning

\section{INTRODUÇÃO}

Aproximadamente um terço dos pacientes internados em Unidades de Terapia Intensiva (UTI) necessita de intubação e instituição de ventilação com pressão positiva. $\mathrm{O}$ manuseio da ventilação nos pacientes que estão se recuperando de insuficiência respiratória aguda, de causa variada, deve buscar dois objetivos: a descontinuação da ventilação mecânica e a remoção da via aérea artificial, tão logo que possível ${ }^{1-3}$.

Diversos estudos já demonstraram que a ventilação mecânica impõe maiores riscos ao paciente, tais como a lesão pulmonar, a pneumonia nosocomial, o trauma da via aérea, a sedação desnecessária e a atrofia muscular respiratória, o que promove o aumento do tempo de ventilação mecânica, da internação e dos custos hospitalares. A pneumonia associada à ventilação mecânica apresenta alta influência na taxa de mortalidade nas UT|2,4-8.

Vale destacar que o processo de desmame do suporte ventilatório é passível de complicações, que vão desde o adiamento desnecessário da extubação traqueal até a ocorrência do risco de complicação secundária pela extubação precoce e a necessidade de re-intubação ${ }^{1,9-11}$. Além disso, os pacientes que necessitam de re-intubação apresentam maiores riscos de morbimortalidade do que aqueles que são extubados com sucesso ${ }^{3,9,12,13}$. Desta forma, é de extrema importância a determinação do modo ideal para a evolução do desmame da ventilação mecânica $(\mathrm{VM})^{5}$.
O processo de descontinuidade da VM e a utilização de medidas eficazes para acelerá-lo têm sido alvo de diversos e extensivos estudos nos últimos anos ${ }^{1,5}$.

Várias estratégias são propostas para determinar a adequada instituição e evolução do desmame ${ }^{14,15}$. A obtenção de parâmetros respiratórios, especialmente quando monitorizados durante o teste de ventilação espontânea, é de grande importância na decisão da equipe clínica ${ }^{4,16}$. A adoção de critérios preditivos para o desmame pode auxiliar na avaliação e na seleção dos pacientes aptos em sustentar a ventilação espontânea imediata e daqueles que necessitam da transição gradual deste suporte ${ }^{17,18}$.

Apesar destas estratégias adotadas durante o desmame serem recomendadas e terem o nível de evidência máxi$\mathrm{ma}^{4}$, nem todos os parâmetros apresentam capacidade preditiva adequada de sucesso na extubação traqueal. Nesse sentido, é imprescindível que o método de avaliação seja simples e reprodutível ${ }^{4,14,19,20}$.

Frente à diversificação dos parâmetros existentes para retirada da prótese ventilatória e dos instrumentos disponíveis para a sua obtenção na prática clínica, é possível que ocorram variações significativas nos métodos e critérios utilizados pelos profissionais atuantes nas UTI.

O objetivo deste estudo foi identificar, caracterizar e demonstrar as práticas utilizadas por fisioterapeutas respiratórios na obtenção dos parâmetros de desmame, bem como a sua execução em UTI, no Distrito Federal.

\section{MÉTODO}

Trata-se de um trabalho descritivo, desenvolvido em diversas Unidades de Terapia Intensiva do Distrito Federal - DF, em 2005, utilizando como instrumento um questionário composto por perguntas objetivas e subjetivas. $\mathrm{O}$ estudo foi aprovado pelo Comitê de Ética em Pesquisa da Universidade Católica de Brasília.

Previamente à aplicação do questionário, foi realizado um levantamento dos hospitais no DF que apresentavam fisioterapeutas nas suas unidades para posterior visita dos pesquisadores. Foram incluídos somente fisioterapeutas formados e com mais de seis meses de atuação em UTI. O questionário usado para essa pesquisa foi publicado por Soo Hoo e Park ${ }^{14}$, traduzido para a língua portuguesa e adaptado pelos autores deste estudo, com o objetivo de adequar o instrumento de pesquisa à realidade da população avaliada. Ele foi auto-aplicável e composto por 31 perguntas objetivas e subjetivas; além disso, não impôs a necessidade de identificação do participante. Esse instrumento continha itens que eram diretamente relacionados ao processo de desmame, tais como o perfil dos 
profissionais que executam essa função e os métodos e critérios mais utilizados.

Os questionários foram distribuídos aos fisioterapeutas de cada hospital selecionado e, após o seu preenchimento, foram recolhidos e tabulados. Em seguida, foi realizado o tratamento estatístico no SPSS 11.0, com descrição da amostra em média $\pm \mathrm{DP}$ e com os valores percentuais das freqüências.

\section{RESULTADOS}

Foram distribuídos 110 questionários e recolhidos 80 devidamente preenchidos, o que correspondeu a mais de $70 \%$ da população de fisioterapeutas atuantes nas UTI no DF. Do total dos fisioterapeutas que responderam completamente os questionários 60 (75\%) eram do sexo feminino e 20 (25\%) do sexo masculino, com média geral de idade de 27,66 \pm 4,39 anos (mínima de 23 e máxima de 45 anos). Em média, a experiência dos fisioterapeutas em terapia intensiva foi de 37,91 \pm 37,64 meses (seis meses no mínimo e 18 anos no máximo), sendo que $90 \%$ são especialistas em cardiorrespiratória ou pneumofuncional.

Esses profissionais eram responsáveis, em média, por sete pacientes em ventilação mecânica por turno de trabalho, o que corresponde a um número adequado de pacientes distribuídos por fisioterapeutas.

A pesquisa realizada entre os fisioterapeutas abrangeu 20 hospitais, os quais podem ser classificados como públicos, privados, especializados, hospitais-escola e um hospital militar. Dentre eles, nove possuíam menos de 100 leitos e 10 de 101 a 499 leitos, o que os classificou em hospitais de médio e grande portes. Somente um hospital possuía mais de 500 leitos.

As UTI eram, em sua maioria (75\%), classificadas como gerais. Onze UTI desses 20 hospitais possuíam 10 leitos ou menos. Outros cinco hospitais variavam de 11 a 24 leitos de UTI e somente quatro deles tinham mais de 25 leitos na UTI.

Em relação ao manuseio diário dos ventiladores mecânicos, foi observado que $98,7 \%$ eram de responsabilidade conjunta dos médicos e fisioterapeutas. Os fisioterapeutas estavam presentes somente pela manhã e tarde em 11 hospitais, até as 23 horas em sete; e, em atendimento 24 horas em outros dois hospitais. Pesquisar o grau de complexidade de cada unidade não foi objetivo do estudo em questão, porém sabe-se que nas UTI de maior complexidades, esses profissionais estiveram presentes também no período noturno.

Diante do questionamento sobre os profissionais responsáveis pelo desmame, foi observado que $61,3 \%$ das respostas destacavam os médicos e fisioterapeutas em conjunto. Aproximadamente $36,3 \%$ das respostas apontaram que a evolução do desmame era feita exclusivamente pelo fisioterapeuta e $2,4 \%$ apenas pelos médicos.

Além disso, em relação às características do desmame da ventilação mecânica e aos métodos adotados, verificou-se que somente 24 fisioterapeutas (30\%) seguiam um protocolo para este fim. Dos 20 hospitais pesquisados, somente seis possuíam protocolo para o desmame, os demais realizavam esse processo baseados na experiência pessoal de cada equipe.

A verificação dos parâmetros para evolução do desmame foi obtida por $83,8 \%$ da população avaliada, pelo menos uma vez ao dia. Esses parâmetros são aferidos no período diurno por $50 \%$ dos profissionais.

Os profissionais foram questionados sobre quais os parâmetros avaliados rotineiramente para possibilitar o início do processo de desmame, sendo que as respostas múltiplas foram permitidas. O parâmetro mais verificado foi a freqüência respiratória (FR), com 98,8\%, seguido por: volume-corrente (VC), com $97,5 \%$ e a saturação periférica de oxigênio $\left(\mathrm{SpO}_{2}\right)$, com $92,5 \%$. Vale ressaltar que foi baixa a percentagem de respostas para a capacidade vital $(13,8 \%)$ e para a gasometria arterial (15\%), que não são utilizadas rotineiramente para avaliar a descontinuidade ou manutenção da ventilação mecânica. Os parâmetros utilizados no questionário estão apresentados na tabela 1.

Tabela 1 - Dados Referentes aos Modos Ventilatórios e Parâmetros Utilizados para o Desmame.

\begin{tabular}{lc}
\hline Modos Ventilatórios & $\mathrm{N}^{\circ}(\%)$ \\
CPAP associado à pressão de suporte & $62(77,5)$ \\
Tubo-T & $44(55)$ \\
Pressão de suporte & $21(26,3)$ \\
SIMV associado à pressão de suporte & $14(17,5)$ \\
CPAP & $8(10)$ \\
SIMV & $4(5)$ \\
Parâmetros de Desmame & \\
Freqüência respiratória & $79(98,8)$ \\
Volume-corrente & $78(97,5)$ \\
Saturação periférica de oxigênio & $74(92,5)$ \\
Freqüência cardíaca & $66(82,5)$ \\
Volume-minuto & $59(73,8)$ \\
Pressão arterial & $56(70)$ \\
FR/VC & $54(67,5)$ \\
Temperatura corpórea & $50(62,5)$ \\
Pimax & $15(18,8)$ \\
Capacidade vital & $11(13,8)$ \\
\hline
\end{tabular}

CPAP: pressão positiva contínua nas vias aéreas; SIMV: ventilação mandatória intermitente sincronizada; FR/VC: índice de respiração rápida e superficial (índice de Tobin); Pimax: pressão inspiratória máxima. 
Em relação às pressões respiratórias máximas (Pimax e Pemax), verificadas por meio de um manovacuômetro, foi verificado que apenas $18,8 \%$ dos fisioterapeutas no DF a realizam rotineiramente (Tabela 1). A tabela 2 mostra informações da maneira como os parâmetros (FR, VC e VE) foram coletados. Eles podem ser mensurados com o auxílio de um ventilômetro, mas também podem ser obtidos por meio da leitura digital nos ventiladores (display). Cerca de 95\% das respostas destacavam a observação do VC por meio do display dos aparelhos, seguido por $76,3 \%$ e $42,5 \%$ das respostas dadas que confirmaram a obtenção do VE e da FR da mesma forma, respectivamente. No entanto, apenas quatro fisioterapeutas (5\%) afirmaram mensurar estes parâmetros utilizando o ventilômetro, levando-se em consideração que somente cinco UTI no DF possuem esse equipamento.

Tabela 2 - Métodos Utilizados para a Mensuração dos Parâmetros de Desmame.

\begin{tabular}{|c|c|}
\hline Métodos & \\
\hline Freqüência respiratória & $\mathrm{N}^{\circ}(\%)$ \\
\hline Display do ventilador & $34(42,5)$ \\
\hline Observação direta & $5(6,3)$ \\
\hline Display do ventilador e observação direta & $27(33,8)$ \\
\hline Leitura do monitor da UTI & $1(1,3)$ \\
\hline $\begin{array}{l}\text { Display do ventilador, observação direta e leitura } \\
\text { do monitor multiparâmetro da UTI }\end{array}$ & $13(16,3)$ \\
\hline \multicolumn{2}{|l|}{ Volume-Corrente } \\
\hline Display do ventilador & $76(95)$ \\
\hline Observação por meio do ventilômetro & $1(1,3)$ \\
\hline $\begin{array}{l}\text { Display do ventilador e observação por meio do } \\
\text { ventilômetro }\end{array}$ & $3(3,8)$ \\
\hline \multicolumn{2}{|l|}{ Volume-Minuto } \\
\hline Display do ventilador & $61(76,3)$ \\
\hline Observação por meio do ventilômetro & $1(1,3)$ \\
\hline Calculado pela multiplicação do VC e FR & $8(10)$ \\
\hline $\begin{array}{l}\text { Display do ventilador e observação por meio do } \\
\text { ventilômetro e cálculo da multiplicação do VC e FR }\end{array}$ & $10(12,6)$ \\
\hline
\end{tabular}

Com relação ao teste de ventilação espontânea, 81,3\% dos fisioterapeutas utilizaram esse recurso, e o tempo de permanência foi, em média, 40,94 $\pm 34,48$ minutos. Verificou-se que somente em dois hospitais as equipes deixavam seus pacientes por duas horas neste teste, os demais mantinham seus pacientes por no mínimo 5 minutos e no máximo por uma hora.

Dentre os modos utilizados para aferir os parâmetros de desmame com vistas à realização da extubação, notou-se que $33,8 \%$ utilizavam o Tubo-T ou a pressão de suporte (PS); $25 \%$ somente a PS; $15 \%$ a pressão positiva contínua nas vias aéreas (CPAP); e apenas
6,3\% utilizavam exclusivamente o Tubo-T. Demais valores são encontrados na tabela 3.

Tabela 3 - Estratégias Utilizadas para a Mensuração dos Parâmetros de Desmame.

\begin{tabular}{lc}
\hline & $\mathrm{N}^{\circ}(\%)$ \\
\hline Tubo-T & $5(6,3)$ \\
CPAP & $12(15)$ \\
Pressão de suporte & $20(25)$ \\
Tubo-T ou CPAP & $8(10)$ \\
Tubo-T ou PS & $27(33,8)$ \\
CPAP/PS & $3(3,8)$ \\
Tubo-T ou CPAP ou PS & $5(6,3)$ \\
\hline
\end{tabular}

CPAP: pressão positiva contínua nas vias aéreas, PS: pressão de suporte

Em relação aos modos ventilatórios mais utilizados, obtiveram-se múltiplas respostas, que podem ser visualizadas na tabela 1. A estratégia mais comum foi a associação da CPAP com a PS $(77,5 \%)$; e a menos utilizada foi a ventilação mandatória intermitente sincronizada (SIMV), com 5\% das respostas.

Com respeito ao questionamento sobre os profissionais responsáveis pela extubação, foram encontradas múltiplas respostas. Aproximadamente $97 \%$ das respostas afirmaram que a extubação é geralmente realizada em conjunto por médicos e fisioterapeutas, sendo que este procedimento é efetivado somente pelos fisioterapeutas em $51 \%$ dos casos. Metade das respostas mostrou que a realização da extubação traqueal é feita até as 17 horas; e 43,8\% apontaram para a extubação independente do período.

\section{DISCUSSÃO}

Com a realização deste estudo foi possível identificar, caracterizar e demonstrar as práticas utilizadas por fisioterapeutas respiratórios na obtenção dos parâmetros de desmame, bem como a sua execução em UTI, no DF. Pode-se observar grande variabilidade nos modos utilizados, na escolha dos parâmetros e na forma como foram coletados. Esse mesmo perfil de diversidade na realização do desmame foi descrito primeiramente no estudo de Soo Hoo e Park ${ }^{14}$, realizado com terapeutas respiratórios em hospitais da cidade de Los Angeles, e posteriormente por Rodrigues e col. ${ }^{21}$, entre os fisioterapeutas de São Paulo.

Assim como ocorre na maioria das UTI, foi observado neste estudo que os responsáveis pela realização do desmame da ventilação mecânica são os médicos e fisioterapeutas em conjunto, e que dos 80 fisioterapeutas, somente $24(30 \%)$ seguiam um protocolo para este 
fim. Alem disso, dos 20 hospitais pesquisados, somente seis possuíam protocolo para o desmame.

Diversas pesquisas têm demonstrado resultados positivos com a implementação de protocolos de desmame realizados por profissionais não médicos ${ }^{22-25}$. No estudo de Ely e col. ${ }^{22}$, os pacientes que foram removidos da ventilação mecânica com a utilização de protocolos específicos para o desmame, permaneceram dois dias a menos nesse processo e tiveram $50 \%$ menos complicações relacionadas ao ventilador. Em outro estudo, Kollef e col. ${ }^{25}$ usaram três diferentes protocolos, também conduzidos por profissionais não médicos, e notaram que o uso do protocolo reduziu a média de duração da ventilação mecânica em 30 horas.

Além disso, o estudo de Walsh e col. ${ }^{17} \mathrm{com}$ mais de 308 pacientes, demonstraram que um simples check-list de alguns parâmetros já poderia predizer se o paciente conseguiria ou não sua independência da ventilação mecânica. Neste sentido, alguns autores questionaram qual a melhor estratégia para a descontinuação da ventilação, porém todos concordaram que a utilização de um protocolo é importante no intuito de evitar atraso desse processo $0^{17,22,23,26}$.

Estudos prévios que investigaram o processo de desmame realizados em UTI em âmbitos nacional e internacional ${ }^{14,21}$, mostraram que a freqüência respiratória e o volume-corrente são os parâmetros mais obtidos no processo de desmame. Esses parâmetros também foram os mais relatados pelos fisioterapeutas no DF. É importante ressaltar a relevância desses dois parâmetros que, quando associados, possibilitam a obtenção do índice de respiração rápida e superficial (FR/VC). Diversos estudos que utilizaram esse índice o apresentaram como

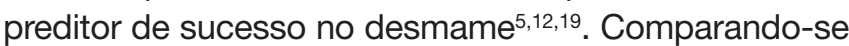
este trabalho com o de Soo Hoo e Park ${ }^{14}$, observa-se que foi esse índice que mostrou maior disparidade, com utilização de $67,5 \%$ e $20 \%$, respectivamente. O estudo realizado por Rodrigues e col. ${ }^{21}$, que verificou o processo de desmame entre fisioterapeutas, realizado em UTI de São Paulo, mostrou valor semelhante (68\%) ao encontrado no presente estudo.

Com relação à pressão inspiratória máxima, pouco utilizada pelos profissionais no DF, foi considerado método de baixa especificidade e grande variabilidade na técnica de coleta $^{19}$, foi um dos parâmetros de desmame largamente utilizado nos hospitais de Los Angeles ${ }^{14}$. As pressões respiratórias máximas (Pimax e Pemax) são analisadas por apenas $18,8 \%$ dos fisioterapeutas respiratórios no DF. Resultado semelhante foi encontrado no estudo realizado nas UTI de São Paulo ${ }^{21}$, onde so- mente $25 \%$ deles realizam essa medida como rotina. Em contrapartida, Soo Hoo e Park ${ }^{14}$ relataram que a maioria (90\%) dos terapeutas entrevistados em seu estudo usava essa medida e, aproximadamente $68 \%$ verificavam a Pimax por três vezes consecutivas, sendo que $75 \%$ dos terapeutas selecionavam como resultado, o maior valor encontrado.

Vale ressaltar que nas UTI no DF, diariamente, cerca de $83,8 \%$ dos fisioterapeutas verificam os parâmetros para evolução do desmame, e que esses parâmetros são aferidos no período diurno por $50 \%$ dos profissionais. No estudo de Soo Hoo e Park ${ }^{14}, 69 \%$ dos terapeutas não declararam em qual período do dia realizavam as coletas dos parâmetros de desmame, mas $31 \%$ responderam que os parâmetros eram obtidos somente no período diurno.

A maioria dos fisioterapeutas no DF realiza a leitura digital nos ventiladores (display) para a mensuração dos parâmetros de desmame (FR, VC e VE). Segundo a literatura, no processo de descontinuação da ventilação mecânica, as medições dos parâmetros devem ser realizadas com o uso de um ventilômetro portátil, já a FR deve ser obtida pela observação direta da caixa torácica ou do ponteiro do ventilômetro analógico ${ }^{19,27}$. No entanto, apenas quatro fisioterapeutas (5\%) afirmaram mensurar estes parâmetros utilizando o ventilômetro, levando-se em consideração que somente cinco UTI no DF possuem esse equipamento. Frente a este fato, deve-se destacar que a obtenção dos parâmetros de desmame pelo display do ventilador pode não apresentar perfeita acurácia, pois aparelhos sem manutenção, descalibrados e a própria adição de pressão positiva podem interferir na precisão da medida ${ }^{21,28}$.

Com relação ao teste de ventilação espontânea, utilizada por mais de $80 \%$ dos fisioterapeutas no DF, que mantêm os pacientes nesse teste, em média, durante 40,94 minutos, a literatura vigente descreve que duas horas não é necessário, bastando alguns minutos após a descontinuação da ventilação para determinar a probabilidade de sucesso ou insucesso no desmame ${ }^{5,28,29}$. Após duas horas em Tubo-T, observa-se elevação no risco de desenvolvimento de complicações como a atelectasia, aumento do trabalho muscular respiratório, retenção de secreção e diminuição da saturação de oxigênio.

Dentre os modos ventilatórios preferencialmente utilizados para aferir os parâmetros de desmame com vistas à realização da extubação, notou-se que, na prática clínica, a maioria dos profissionais avalia os parâmetros com o paciente ainda conectado à ventilação mecânica. As pesquisas realizadas descreveram que 
os parâmetros devem ser coletados com o paciente em ventilação espontânea com o Tubo- $T^{16,19,27}$, pois, nesta situação, os parâmetros que representam a resposta do sistema respiratório são reais e fidedignos, o que nem sempre acontece quando a coleta é feita com o paciente conectado à ventilação mecânica ${ }^{30}$.

Alem disso, foram investigados os modos ventilatórios mais utilizados para a evolução do desmame. Notouse que a maioria dos fisioterapeutas no DF $(77,5 \%)$ elegeu a associação da CPAP com a PS como a estratégia mais comum para a progressão do desmame e a menos utilizada foi a SIMV (5\%). Neste sentido, já está bem definido na literatura que o uso da PS é superior ao uso do modo SIMV nesse processo, sendo, portanto, considerado que o SIMV é um modo desfavorável para o desmame, uma vez que, comprovadamente, aumenta o tempo de permanência em suporte ventilatório ${ }^{15,31,32}$.

Vários estudos enfatizam a importância da avaliação dos parâmetros de desmame da ventilação mecânica baseados em evidências. Contudo, a maioria dos parâmetros, quando avaliados isoladamente, é falha como preditor de sucesso ou insucesso no desmame e na extubação. Assim, esses parâmetros devem sempre estar associados, para maior segurança e confiabilidade para a tomada de decisão $2,5,19,29$.

\section{CONCLUSÃO}

O detalhamento do método de desmame é fundamental, visto que o seu sucesso depende da técnica escoIhida e, principalmente, da forma como é desenvolvido e experimentado pela equipe.

Observou-se grande variabilidade nos modos utilizados, na escolha dos parâmetros, e na forma com que foram coletados os dados para a realização do desmame, sugerindo, então, a falta de rotina nos serviços e a necessidade de implantação de protocolos simples e facilmente aplicáveis na realização desse processo.

$\mathrm{Na}$ atualidade, a adoção de um consenso, de protocolos e do detalhamento do método utilizado, seja ele qual for, é fundamental, visto que o despreparo e a imperícia na assistência ventilatória podem levar ao insucesso qualquer método de desmame, gerando comprometimento importante na evolução do paciente.

\section{REFERÊNCIAS}

01. Alia I, Esteban A - Weaning from mechanical ventilation. Crit Care, 2000;4:72-80.

02. II Consenso Brasileiro de Ventilação Mecânica. J Pneumol, 2000;26:54-60.

03. Maclntyre NR - Evidence-based ventilator weaning and discontinuation.
Respir Care, 2004;49:830-836.

04. Evidence-based guidelines for weaning and discontinuing ventilatory support. A collective task force facilitated by the American College of Chest Physicians, the American Association for Respiratory Care, and the American College of Critical Care Medicine. Respir Care, 2002;47(1):69-90.

05. Epstein SK - Extubation. Respir Care, 2002;47:483-495.

06. Colice GL, Stukel TA, Dain B - Laryngeal complication of prolonged intubation. Chest, 1989;96:877-884.

07. Fagon JY, Chastre J, Hance AJ et al - Nosocomial pneumonia in ventilated patients: a cohort study evaluating attributable mortality and hospital stay. Am J Med, 1993;94:281-288.

08. Heyland DK, Cook DJ, Griffith L et al - The attributable morbidity and mortality of ventilator-associated pneumonia in the critically ill patient. The Canadian Critical Trials Group. Am J Respir Crit Care Med, 1999;159:1249-1256.

09. Esteban A, Frutos-Vivar F, Ferguson ND et al - Noninvasive positivepressure ventilation for respiratory failure after extubation. N Engl J Med, 2004;350:2452-2460.

10. Manthous CA - The anarchy of weaning techniques. Chest, 2002;121:1738-1740.

11. Gil B, Frutos-Vivar F, Esteban A - Deleterious effects of reintubation of mechanically ventilated patients. Clin Pulm Med, 2003;10:226-230.

12. Epstein SK, Ciubotaru RL, Wong JB - Effect of failed extubation on the outcome of mechanical ventilation. Chest, 1997;112:186-192.

13. Epstein SK, Ciubotaru RL - Independent effects of etiology of failure and time to reintubation on outcome for patients failing extubation. Am J Respir Crit Care Med, 1998;158:489-493.

14. Soo Hoo GW, Park L - Variations in the measurement of weaning parameters: a survey of respiratory therapists. Chest, 2002;121:1947-1955.

15. Manthous CA, Schmidt GA, Hall JB - Liberation from mechanical ventilation: a decade of progress. Chest, 1998;114:886-901.

16. Khamiees M, Raju P, DeGirolamo A et al - Predictors of extubation outcome in patients who have successfully completed a spontaneous breathing trial. Chest, 2001;120:1262-1270.

17. Walsh TS, Dodds S, McArdle F - Evaluation of simple criteria to predict successful weaning from mechanical ventilation in intensive care patients. Br J Anaesth, 2004;92:793-799.

18. Esteban A, Alia I, Gordo F et al - Extubation outcome after spontaneous breathing trials with t-tube or pressure support ventilation. Am J Respir Crit Care Med, 1997;156:459-465

19. Yang KL, Tobin MJ - A prospective study of indexes predicting the outcome of trials of weaning from mechanical ventilation. $\mathrm{N}$ Engl $\mathrm{J}$ Med, 1991;324:1445-1450.

20. Yang KL - Reproducibility of weaning parameters. A need for standardization. Chest, 1992;102:1829-1832.

21. Rodrigues MM, Fiore JF, Benassule E et al - Variações na mensuração dos parâmetros de desmame da ventilação mecânica em hospitais da cidade de São Paulo. RBTI, 2005;17:28-32.

22. Ely EW, Meade MO, Haponik EF et al - Mechanical ventilator weaning protocols driven by nonphysician health-care professionals: evidence-based clinical practice guidelines. Chest, 2001;120:(Suppl6):454S-463S.

23. Marelich GP, Murin S, Battistella F et al - Protocol weaning of mechanical ventilation in medical and surgical patients by respiratory care practitioners and nurses: effect on weaning time and incidence of ventilator associated pneumonia. Chest, 2000;118:459-467.

24. Scheinhorn DJ, Chao DC, Stearn-Hassenpflug $M$ et al - Outcomes in post-ICU mechanical ventilation: a therapist-implemented weaning protocol. Chest, 2001;119:236-242.

25. Kollef $\mathrm{MH}$, Shapiro $\mathrm{SD}$, Silver $\mathrm{P}$ et al - A randomized, controlled trial of protocol-directed versus physician-directed weaning from mechanical ventilation. Crit Care Med, 1997;25:567-574

26. Keenan SP - Weaning protocols: here to stay. Lancet, 2002;359:186-187.

27. Epstein SK - Etiology of extubation failure and the predictive value of the rapid shallow breathing index. Am J Respir Crit Care Med, 1995;152:545-549.

28. Esteban A, Frutos F, Tobin MJ et al - A comparison of four methods of weaning patients from mechanical ventilation. N Eng J Med, 1995;332:345-350.

29. Conti G, Montini L, Pennisi MA et al - A prospective, blinded evaluation of indexes proposed to predict weaning from mechanical ventilation. Intensive Care Med, 2004;30:830-836.

30. Lee KH, Hui KP, Chan TB et al - Rapid shallow breathing (frequency-tidal volume ratio) did not predict extubation outcome. Chest, 1994;105:540-543.

31. Ely EW, Baker AM, Dunagan DP et al - Effect on the duration of mechanical ventilation of identifying patients capable of breathing spontaneously. New Eng J Med, 1996;335:1864-1869.

32. HessD-Ventilatormodesusedinweaning.Chest,2001;120:(Suppl6):474S$476 S$. 